\title{
Spontaneous corpectomy and anterior arthrodesis in lumbar spine: how Ankylosing Spondylitis can resolve a vertebral fracture
}

\author{
Daniele Vanni ${ }^{1}$, Andrea Pantalone ${ }^{1}$, Stefano Di Carlo ${ }^{1}$, Vincenzo Magliani ${ }^{2}$, Pedro Berjano ${ }^{3}$, Vincenzo Salini ${ }^{1}$ \\ ${ }^{1}$ Orthopaedic and Traumatology Department, “G. D’Annunzio” University, Chieti, Italy; ${ }^{2}$ Neurotraumatology and Vertebro-Medullary Surgery \\ Department, Renzetti Hospital, Lanciano, Italy; ${ }^{3}$ IVth Spine Division, IRCCS Istituto Ortopedico Galeazzi, Milan, Italy \\ Correspondence to: Daniele Vanni. Orthopaedic and Traumatology Department, “G. D’Annunzio” University, Chieti, Italy. \\ Email: danielevannimd@libero.it.
}

\begin{abstract}
Between the $80 \%$ and $90 \%$ of vertebral fractures in over 65 years people are due to osteoporosis. Over the $30 \%$ of patients affected by vertebral osteoporosis fractures needs of surgical treatment and the $12 \%$ presents complications requiring an invasive surgical approach. We report an unusual case of spontaneous corpectomy and anterior arthrodesis occurred in a patient suffering from Ankylosing Spondylitis. This event invites us to reflect about the magnitude of the biological power of the bone healing, even in adverse conditions.
\end{abstract}

Keywords: Corpectomy; anterior arthrodesis; Ankylosing Spondylitis; lumbar spine

Submitted Oct 05, 2016. Accepted for publication Jan 12, 2017.

doi: 10.21037 /jss.2017.02.02

View this article at: http://dx.doi.org/10.21037/jss.2017.02.02

\section{Introduction}

Ankylosing spondylitis is a form of arthritis that primarily affects the spine. It causes inflammation of the spine joints that can lead to severe and chronic pain. In advanced cases, the inflammation process can lead to new bone formation, in order to fuse the spine in a fixed and immobile position, causing sometimes a forward-stooped posture (1). Osteoporosis is a systemic skeletal disease marked by a low bone mass and a microarchitectural deterioration of the bone tissue, leading to an increased fragility, especially in the hip, spine and wrist (2). Between $80 \%$ and $90 \%$ of vertebral fractures in over 65 years people are due to osteoporosis, but only the $30 \%$ are clinically manifest. Degenerative disc disease and senile neuromotor and neurosensory decay, are associated with a progression of the kyphosis of the spine, resulting in an anterior translation of the gravitational axis and a progressive posterior ligaments distraction. Over the $30 \%$ of the patients affected by vertebral osteoporosis fractures needs of surgical treatment and the $12 \%$ of them presents complications that require an invasive surgical approach. In most cases these are pathologic fractures, that may occur spontaneously or after a minor trauma (3).

\section{Case presentation}

An 82-year-old Caucasian male went to the emergency department due to a low back pain and a left hip pain. The patient reported to be suffering from Ankylosing Spondylitis. The physical examination showed pain at the lumbar spine associated with contracture of the lumbar paraspinal muscles, bilaterally. The left leg appeared shortened, flexed and externally rotated. There were not significant neurological deficits. The X-ray of the spine, routinely performed, documented a deformity of L1 and L2 (Figure 1). The CT scan allowed to deeply analyze this two deformities that appeared as two old and stabilized vertebral fractures. In fact the patient reported that he fallen from a tree about 17 years earlier. Therefore, without any pharmacological, conservative or surgical treatment, the patient experienced a spontaneous vertebral fracture healing. In fact a spontaneous corpectomy of $\mathrm{L} 1$, whose soma was reabsorbed itself over time and an anterior arthrodesis D12-L2 occurred, 


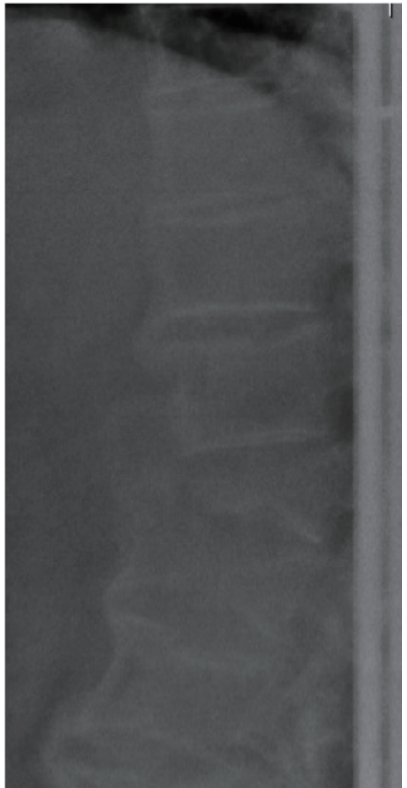

Figure $1 \mathrm{Rx}$ evaluation.

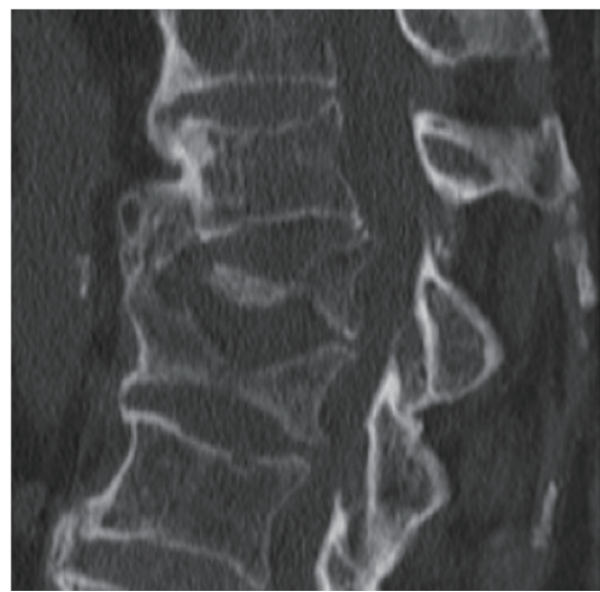

Figure 2 CT evaluation.

although the soma of L2 had also reported a fracture (Figures 2-4).

\section{Discussion}

We report this unusual case that demonstrates how the biology spontaneously acts to solve alone what the man would do in an artificial way. The fracture of the body induces an height soma reduction and an increase of the kyphosis of the spine, especially at the level of the anterior

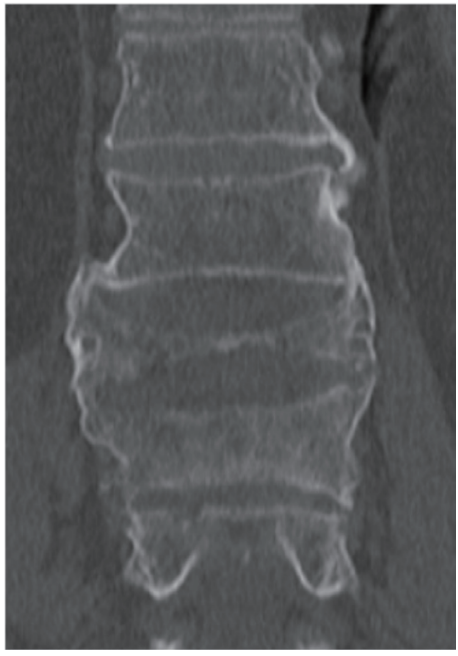

Figure 3 CT evaluation.

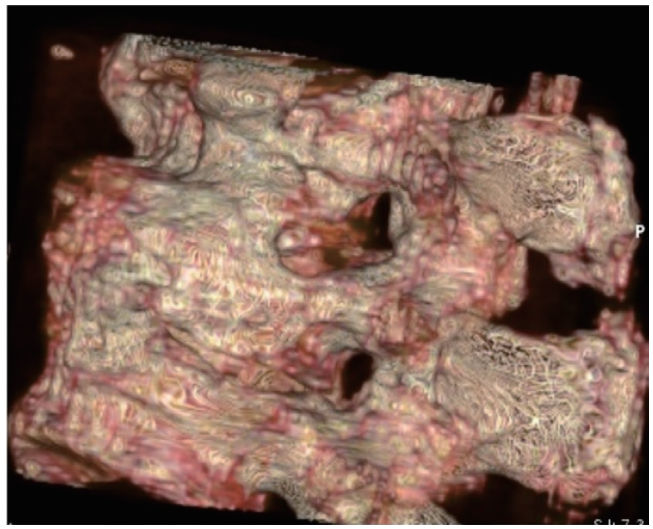

Figure 4 3D CT evaluation.

spinal column. The more involved vertebrae are $\mathrm{T} 7$ and T8, in the middle thoracic column and T12 and L1 in the thoracolumbar transition where the great flexion occurs. The thoracolumbar hinge is most subjected to the stress, because in this zone there is the inversion of two curves with an opposite convexity. The progression of the kyphosis and anterior displacement of the gravity axes lead to an increase in the anterior flexion, producing a new fracture. In fact the risk of another vertebral fracture increases 5-fold after the first event ('domino-effect'). These conditions must be considered in case of an open approach, especially in order to identify the correct level to treat and to select the appropriate surgery instrumentation, because in this cases there is and high risk of fixation failure (4-6). In this case, probably, the ability of the vertebral body to fuse 
itself, induced by the Ankylosing Spondylitis, prevailed on the worsening of the fracture, reaching a balance and obtaining a natural resolution. Therefore, the surgical treatment of this type of fractures might result complex and difficult, even for experienced surgeons $(7,8)$. In some cases the progression of kyphosis and the poor bone stock require corpectomies and vertebral fixations. These approaches could be very dangerous, due to also a high risk of intraoperative blood loss. However, it is the most correct treatment, especially from a biomechanical point of view.

\section{Conclusions}

To our knowledge, founded on a review of the literature, other cases of spontaneous corpectomy and anterior arthrodesis have never been reported until now. This event invites us to reflect about the biological capabilities of the bone healing, even in adverse conditions. In fact in this case the vertebrae were able to find a solution and to achieve the stability.

\section{Acknowledgements}

None.

\section{Footnote}

Conflicts of Interest: The authors have no conflicts of interest to declare.

Informed Consent: Written informed consent was obtained from the patient for publication of this manuscript and any accompanying images.

Cite this article as: Vanni D, Pantalone A, Di Carlo S, Magliani V, Berjano P, Salini V. Spontaneous corpectomy and anterior arthrodesis in lumbar spine: how Ankylosing Spondylitis can resolve a vertebral fracture. J Spine Surg 2017;3(1):73-75. doi: 10.21037/jss.2017.02.02

\section{References}

1. Altun I, Yuksel KZ. Ankylosing Spondylitis: Patterns of Spinal Injury and Treatment Outcomes. Asian Spine J 2016;10:655-62.

2. Augat $\mathrm{P}$, Weyand D, Panzer S, et al. Osteoporosis prevalence and fracture characteristics in elderly female patients with fractures. Arch Orthop Trauma Surg 2010;130:1405-10.

3. Christiansen BA, Bouxsein ML. Biomechanics of vertebral fractures and the vertebral fracture cascade. Curr Osteoporos Rep 2010;8:198-204.

4. Uchida K, Kobayashi S, Nakajima H, et al. Anterior expandable strut cage replacement for osteoporotic thoracolumbar vertebral collapse. J Neurosurg Spine 2006;4:454-62.

5. Graillon T, Farah K, Rakotozanany P, et al. Anterior approach with expandable cage implantation in management of unstable thoracolumbar fractures: Results of a series of 93 patients. Neurochirurgie 2016;62:78-85.

6. Theologis AA, Tabaraee E, Toogood P, et al. Anterior corpectomy via the mini-open, extreme lateral, transpsoas approach combined with short-segment posterior fixation for single-level traumatic lumbar burst fractures: analysis of health-related quality of life outcomes and patient satisfaction. J Neurosurg Spine 2016;24:60-8.

7. Berjano P, Lamartina C. Minimally invasive lateral transpsoas approach with advanced neurophysiologic monitoring for lumbar interbody fusion. Eur Spine J 2011;20:1584-6.

8. Berjano P, Blanco JF, Rendon D, et al. Finite element analysis and cadaveric cinematic analysis of fixation options for anteriorly implanted trabecular metal interbody cages. Eur Spine J 2015;24 Suppl 7:918-23. 